\title{
microRNA-513c suppresses the proliferation of human glioblastoma cells by repressing low-density lipoprotein receptor-related protein 6
}

\author{
JIAJUN XU ${ }^{1 *}$, TAO SUN ${ }^{2 *}$ and XUEBING $\mathrm{HU}^{3}$ \\ ${ }^{1}$ Department of Neurosurgery, Shandong Provincial Hospital Affiliated to Shandong University, Shandong University, Jinan, \\ Shandong 250021; ${ }^{2}$ Department of Neurosurgery, Zaozhuang Hospital of Zaozhuang Coal Mining Group, Zaozhuang, \\ Shandong 277101; ${ }^{3}$ Department of Neurosurgery, Tengzhou Workers Hospital, Tengzhou, Shandong 277500, P.R. China
}

Received July 23, 2014; Accepted April 14, 2015

DOI: $10.3892 / \mathrm{mmr} .2015 .3913$

\begin{abstract}
Aberrant activation of the $\mathrm{Wnt} / \beta$-catenin signaling pathway is frequently observed in glioblastoma (GBM) cells. Therefore, it was hypothesized that low-density lipoprotein receptor-related protein 6 (LRP6) may be involved in activating the $\mathrm{Wnt} / \beta$-catenin pathway in the progression of GBM. The present study reported that the expression of microRNA (miR)-513c was markedly downregulated in GBM cells and GBM tissues compared with that in normal human astrocytes and normal brain tissues. Previous studies have demonstrated that miR-513c is critical in a variety of biological processes in various human cancer cells. The role of this $\mathrm{miR}$ in GBM cells was therefore investigated in the present study. Ectopic expression of miR-513c reduced the proliferation and anchorage-independent growth of GBM cells, whereas inhibition of miR-513c promoted this effect. Bioinformatic analysis further identified LRP6, a putative tumor suppressor, as a potential target of miR-513c. Luciferase reporter assays revealed that miR-513c directly bound to the 3'-untranslated region of LRP6 mRNA and repressed the expression at the transcriptional as well as the translational level. In functional assays, miR-513c suppressed GBM cell proliferation, which was reversed by an inhibitor of miR-513c. In conclusion, the present study provided compelling evidence that miR-513c functions as a tumor suppressor miRNA, which may be important in the inhibition of cell proliferation in GBM. In addition, the tumor suppressive effects were mediated predominantly through the direct suppression of the expression of LRP6.
\end{abstract}

Correspondence to: Dr Jiajun Xu, Department of Neurosurgery, Shandong Provincial Hospital Affiliated to Shandong University, Shandong University, 324 Jingwu Road, Jinan, Shandong 250021, P.R. China

E-mail: jiajunxushandong@163.com

"Contributed equally

Key words: microRNA-513c, human glioblastoma, low-density lipoprotein receptor-related protein 6 , cell proliferation

\section{Introduction}

Glioblastoma (GBM) is the most common and most lethal type of brain tumor in adults (1). The current treatment for GBM involves resection, followed by radiation and chemotherapy. Therefore, there is an urgent requirement to elucidate the underlying molecular mechanisms of GBM and identify novel molecular targets for the treatment of this disease.

MicroRNAs (miRs) are endogenous non-coding small RNAs, which suppress genes by triggering either mRNA degradation or translational repression by binding to the 3'-untranslated region (3'-UTR) of target mRNA (2-4). miRNAs were confirmed to be involved in several processes, including cell proliferation, apoptosis, invasion and differentiation in various types of cancer $(4,5)$. The Wnt signaling pathway is important in tumorigenesis. The low-density lipoprotein (LDL) receptor-related protein-6 (LRP6), a novel member of the expanding LDL receptor family, is a cell surface receptor and is critical in the activation of the canonical $\mathrm{Wnt} / \beta$-catenin signaling pathway (6-8). The underlying mechanism of the tumor suppressive function of miR-513 as well as its target genes have remained to be elucidated. The present study identified and investigated the targeted regulation of LRP6 by miR-513c in GBM cells and assessed its function in cell proliferation.

\section{Materials and methods}

Clinical specimens. Human GBM tissues $(\mathrm{n}=8)$ and normal brain tissues were obtained from patients with GBM at Shandong Provincial Hospital affiliated to Shandong University (Jinan, China). The present study was approved by the ethics committee of the Shandong Provincial Hospital Affiliated to Shandong University, Shandong University (Jinan, China) and written informed consent was obtained from all patients. Tissue samples were collected at surgery, immediately frozen in liquid nitrogen and stored until the total RNAs or proteins were extracted.

Cell culture. The human GBM cell lines LN319, SNB19, LN18, U251MG, U118MG, T98G, U373MG and LN382T were purchased from the American Type Culture Collection 
(Manassas, VA, USA) and grown in Dulbecco's modified Eagle's medium (Gibco-BRL, Invitrogen Life Technologies, Carlsbad, CA, USA), supplemented with $10 \%$ fetal bovine serum (FBS; Sigma-Alrich, St. Louis, MO, USA) and 100 units/ml penicillin-streptomycin (Invitrogen Life Technologies). Normal human astrocytes (NHAs) were obtained from Lonza (Walkersville, MD, USA) and cultured in the provided astrocyte growth media, supplemented with recombinant human epidermal growth factor (Robustnique Corporation Ltd., Tianjin, China), insulin (Sigma-Aldrich), ascorbic acid (Sigma-Aldrich), GA-1000 (Sigma-Aldrich), L-glutamine (Sigma-Aldrich) and 5\% FBS. All cell lines were cultured in a humidified incubator at $37^{\circ} \mathrm{C}$ in an atmosphere of $5 \% \mathrm{CO}_{2}$ and $95 \%$ air.

Plasmids, small interfering (si)RNA and transfection. For ectopic expression of LRP6, the LRP6 open reading frame with a 3'-UTR was amplified using polymerase chain reaction (PCR) and sub-cloned into p-enhanced green fluorescent protein-N3 (Invitrogen Life Technologies). To construct a luciferase reporter vector, the LRP6 3'-UTR fragment, containing putative binding sites for $\mathrm{miR}-513 \mathrm{c}$, was amplified using PCR and cloned downstream of the luciferase gene in the pGL3-luciferase reporter plasmid (Promega Corporation, Madison, WI, USA). The constructs were verified by sequencing. The miR-513c mimics (HmiR0671), negative control (NC) and miR-513c inhibitor (HmiR-AN0568) were purchased from Genecopoeia Co. (Rockville, MD, USA) and transfected into glioblastoma cells using Lipofectamine 2000 reagent (Invitrogen Life Technologies) according to the manufacturer's instructions.

For LRP6 depletion, small interfering (si)RNA was synthesizedandpurified by RiboBioCo.,Ltd.(Guangzhou,Guangdong, China). The LRP6-siRNA sequences used were as follows: LRP6-siRNA ${ }^{\#}$ 1, 5'-CCGATGCAATGGAGATGCAAA-3' and LRP6-siRNA ${ }^{\# 2}$, 5'-CGCACTACATTAGTTCCAAAT-3'. The transfection of siRNAs was performed using Lipofectamine 2000 (Invitrogen Life Technologies) according to the manufacturer's instructions.

$R N A$ extraction and reverse transcription quantitative $(R T-q) P C R$. For miRNA quantification, the total RNA, including miR, was extracted from cultured cells and patient samples using the mirVana miRNA Isolation kit (Ambion, Austin, TX, USA), according to the manufacturer's instructions. The cDNA was synthesized from $5 \mathrm{ng}$ total RNA using the Taqman miRNA reverse transcription kit (Applied Biosystems, Thermo Fisher Scientific, Waltham, MA, USA). The expression levels of miR-513c were quantified using the miRNA-specific TaqMan miRNA Assay kit (Applied Biosystems). The relative expression levels of miR-513c following normalization to U6 small nuclear RNA were calculated using $2^{-[\mathrm{Ct}(\mathrm{miR}-513 \mathrm{c})-\mathrm{Ct}(\mathrm{U} 6)]}$. qPCR was performed using an SYBR kit (Qiagen, Shanghai, China) on a Light Cycler system (LightCycler480; Roche Diagnostics, Basel, Switzerland). The primers selected were as follows: LRP6 forward, 5'-CCTCAATGCGATTTGTTCC-3' and reverse, 5'-GGTGTCAAAGAAGCCTCTGC-3'; cyclin D1 forward, 5'-TCCTCTCCAAAATGCCAGAG-3' and reverse, 5'-GGCGGATTGGAAATGAACTT-3'; Мус forward, 5'-TCAAGAGGCGAACACACAAC-3' and reverse, 5'-GGCCTTTTCATTGTTTTCCA-3'; and LEF1 forward, 5'-CACTGTAAGTGATGAGGGGG-3' and reverse, 5'-TGGATCTCTTTCTCCACCCA-3'.

The expression was normalized against the geometric mean of GAPDH to control the variability in expression levels (GAPDH forward, 5'-GACTCATGACCACAGTCCATGC-3' and reverse, 3'-AGAGGCAGGGATGATGTTCTG-5') and was calculated as $2^{-[\mathrm{Ct}(\mathrm{LRP} 6 / \mathrm{cyclinD1} / \mathrm{Myc} / \mathrm{LEF} 1)-\mathrm{Ct}(\mathrm{GAPDH})]}$.

MTT and colony formation assays. Cell growth was measured using an MTT assay. The SNB19 cells were seeded into 96-well plates in medium containing $10 \%$ FBS at 3,000 cells/well. For quantitation of cell viability, the cultures were stained after 1, 2, 3 and 4 days with MTT. Briefly, $20 \mu 15 \mathrm{mg} / \mathrm{ml}$ MTT solution (Sigma-Aldrich) was added to each well followed by incubation at $37^{\circ} \mathrm{C}$ for $4 \mathrm{~h}$. The medium was then removed from each well and the resulting MTT formazan was solubilized in $150 \mu \mathrm{l}$ dimethylsulfoxide (Sigma-Aldrich). The absorbance at $490 \mathrm{~nm}$ was measured using a Multiskan plate reader (Thermo Fisher Scientific).

For colony formation assays, the SNB19 cells were seeded into three $6-\mathrm{cm}$ cell culture dishes $\left(1 \times 10^{3}\right.$ cells/well) and incubated for 10 days in medium containing $10 \%$ FBS. The colonies were washed with phosphate-buffered saline and stained with $1 \%$ crystal violet for $30 \mathrm{sec}$ following fixation with $10 \%$ formaldehyde (Beyotime Institute of Biotechnology, Shanghai, China) for $15 \mathrm{~min}$. The number of colonies, defined as $>50$ cells/colony, were counted. Three independent experiments were performed and the results were statistically analyzed using a paired t-test.

Anchorage-independent growth assay. The cells were trypsinized and 1,000 cells were re-suspended in $2 \mathrm{ml}$ complete medium containing 0.3\% agar (Sigma-Aldrich). The agar-cell mixture was seeded on top of a bottom layer, consisting of $1 \%$ agar in complete medium. The plates were incubated at $37^{\circ} \mathrm{C}$ in a humid atmosphere of $5 \% \mathrm{CO}_{2}$ for 2 weeks until colony formation. The colonies were stained with $0.5 \%$ crystal violet for counting colony number under an inverted fluorescence microscope (Motic AE30; Microscope Systems Ltd., Glasgow, UK) and images of cell colonies were captured at magnification of $x 100$. Only cell colonies containing $>50$ cells were counted. The experiment was performed three independent times for each cell line.

Luciferase assays. The cells were seeded in triplicate in 24 -well plates $\left(5 \times 10^{4} /\right.$ well) and cultured for $24 \mathrm{~h}$. The pGL3-luciferase reporter gene plasmid, pGL3-LRP6-3'-UTR, or the control-luciferase plasmid were co-transfected into the cells with the control pRL-TK Renilla plasmid (Promega Corporation) using Lipofectamine 2000 reagent (Invitrogen Life Technologies). Luciferase and Renilla activities were assayed $48 \mathrm{~h}$ following the transfection by lysing the cells with Cell Lysis solution (Beyotime Institute of Biotechnology) and detecting the fluorescence intensity using the dual luciferase assay kit.

Western blot analysis. The protein lysates were prepared and equal quantities $(40 \mu \mathrm{g})$ of protein were subjected to 
$10 \%$ SDS-PAGE. The proteins were transferred onto polyvinylidene fluoride membranes for $2 \mathrm{~h}$ at $4^{\circ} \mathrm{C}$, at a current of $125 \mathrm{~mA}$. The membranes were blocked with $5 \%$ non-fat milk (Beyotime Institute of Biotechnology) for $2 \mathrm{~h}$ and were subsequently incubated overnight with anti-LRP6 (\#3395), anti-cyclin D1 (\#2978), anti-C-Myc (\#5605), anti-LEF1 (\#2230) (1:1,000; Cell Signaling Technology, Inc., Danvers, MA, USA), anti-p84 (ab487; 1:2,000; Abcam, Cambridge, UK) and $\alpha$-tubulin (1:2,000; Sigma-Aldrich), which was used as a reference protein. Following antibody incubation, the membranes were washed with Tris-buffered saline containing Tween-20 (TBST; Sigma-Aldrich) three times for 5 min each time. Following washing with TBST and incubation with either anti-rabbit horseradish peroxidase-conjugated secondary antibody (A0545; 1:5,000; Sigma-Aldrich) for $2 \mathrm{~h}$ at room temperature, immunocomplexes were visualized using the ECL Advanced Western Blotting Detection kit (GE Healthcare, Little Chalfont, UK) according to the manufacturer's instructions.

Statistical analysis. All statistical analyses were performed using SPSS 19.0 (International Business Machines, Armonk, NY, USA). Student's t-test was used to determine the significance of the differences between the two groups of data in all the pertinent experiments. $\mathrm{P}<0.05$ was considered to indicate a statistically significantly difference.

\section{Results}

Expression of miR-513c is downregulated in GBM tissues and cell lines. To investigate the role of miR-513c in the development of GBM, the expression levels of miR-513c in GBM tissues and GBM cell lines were assessed by RT-qPCR (Fig. 1A). The results demonstrated that the expression levels of miR-513c were consistently lower in the GBM tissues compared with those in the normal brain tissues. In addition, in all eight GBM cell lines, significantly lower expression levels of miR-513c were observed compared with those in the NHAs. These results suggested that miR-513c is significantly reduced in GBM and may serve as a prognostic marker for patients with GBM.

miR-513c inhibits GBM cell proliferation. Since miR-513c was significantly downregulated in GBM tissues and cell lines, the present study investigated whether miR-513c exhibits a tumor suppressive role in the cell proliferation of GBM. SNB19 cells were transfected with miR-513c mimics, miR-513c inhibitor or the respective controls. The relative expression levels of miR-513c were confirmed using RT-qPCR (Figs. 2A and 3A). The effect of miR-513c on cell proliferation was determined using MTT, colony formation and anchorage-independent growth assays. To investigate the role of upregulation of $\mathrm{miR}-513 \mathrm{c}$ in the development and progression of GBM, its effect on cellular proliferation was assessed. An MTT assay demonstrated that miR-513c-transduced SNB19 cells exhibited significantly lower growth rates compared with those in the control cells at day four after seeding (Fig. 2B). The colony formation assays consistently revealed that enforced expression of miR-513c significantly reduced the number of colonies of SNB19 cells following 14 days of culture compared with

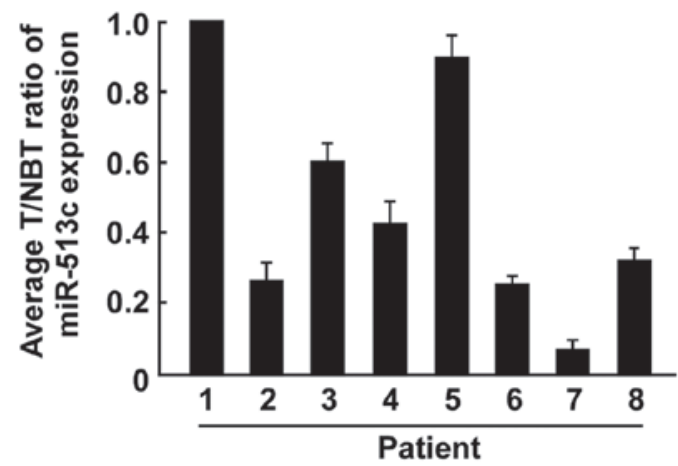

B

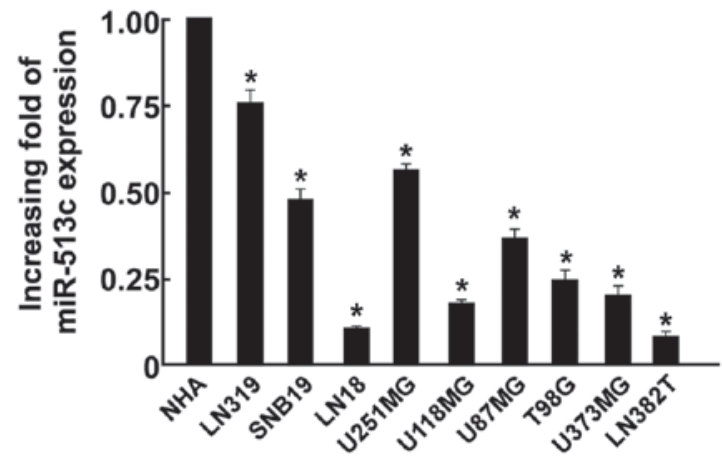

Figure 1. Expression of miR-513c in human GBM tissues and cell lines. (A) Relative expression levels of miR-513c in eight paired primary GBM tissues and the matched normal brain tissue from patients were detected by RT-qPCR analysis. (B) RT-qPCR analysis of the expression of miR-513c in GBM cell lines, including LN319, SNB19, LN18, U251MG, U118MG, T98G, U373MG and LN382T. All experiments were repeated at least three times and values are expressed as the mean of three independent experiments ("P<0.05 vs. NHA). GBM, glioblastoma; RT-qPCR, reverse transcription quantitative polymerase chain reaction; T/NBT, tumor/normal brain tissue; miR, microRNA; NHA, normal human astrocytes.

that in the control group (Fig. 2C). Of note, it was demonstrated that enforced expression of miR-513c in SNB19 cells significantly decreased their anchorage-independent growth ability (Fig. 2D). By contrast, the cell growth rates and colony numbers of SNB19 cells transfected with the miR-513c inhibitor (miR-513c-in) were significantly higher compared with those transfected with the NC (Fig. 3B and C). In addition, the anchorage-independent growth ability of SNB19 cells was significantly increased in following transfection with miR-513c-in (Fig. 3D). These results demonstrated that miR-513c reduced GBM cell tumorigenicity in vitro.

miR-513c directly targets LRP6 by binding to its 3 '-UTR. It is generally accepted that miRNAs exert their functions by regulating the gene expression of their downstream target genes (2-4). Bioinformatic analysis was used to search for the potential regulatory targets of miR-513c. LRP6, a tumor suppressor gene containing a binding site for miR-513c, was selected as the target gene for further analysis (Fig. 4A).

To determine whether miR-513c affected the expression of LRP6, the expression levels of LRP6 were detected in the SNB19 cells, which were transfected with miR-513c mimics, miR-513c-in or the respective controls. Western blot analysis demonstrated that the miR-513c mimics markedly suppressed the protein expression of LRP6 in SNB19 cells (Fig. 4B), 
A

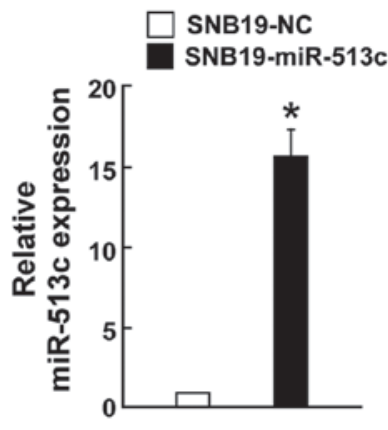

C

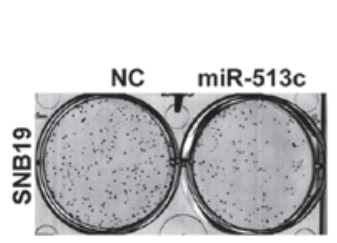

NC $\square_{\text {miR-513c }}$

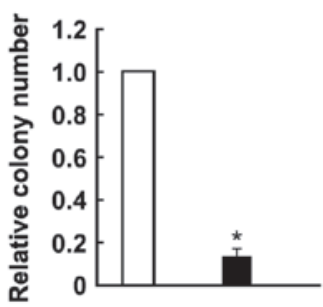

B

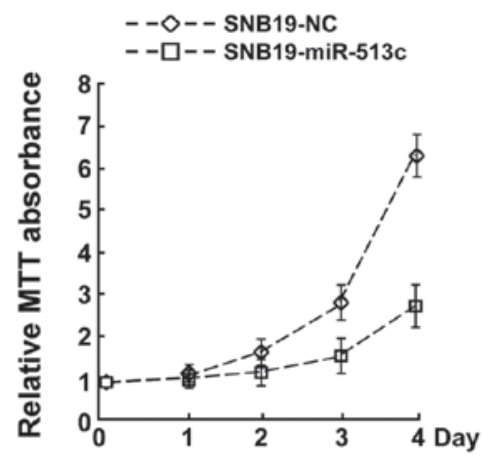

D
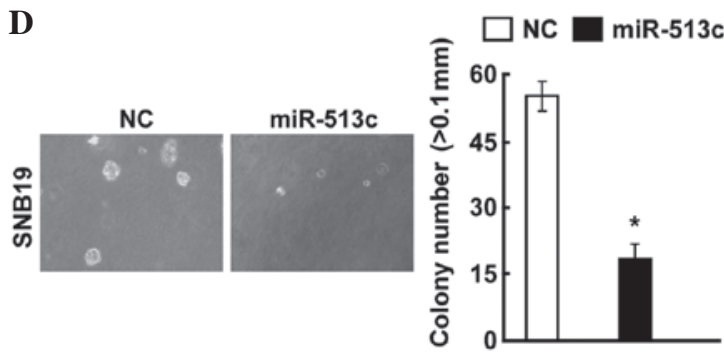

Figure 2. miR-513c upregulation inhibits GBM cell proliferation. (A) Confirmation of the expression of miR-513c following transfection by reverse transcription quantitative polymerase chain reaction analysis. (B) MTT assays revealed that the upregulation of miR-513c inhibited the growth of the SNB19 GBM cell line. (C) Representative micrographs (left) and quantification (right) of crystal violet-stained cell colonies (magnification, $\mathrm{x} 4$ ). (D) Upregulation of miR-513c impaired the anchorage-independent growth of SNB19 cells. Representative micrographs (left) and quantification of colonies, which were $>0.1 \mathrm{~mm}$ (right)

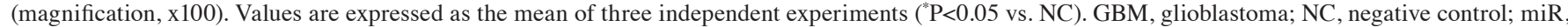
microRNA; NC, normal control.

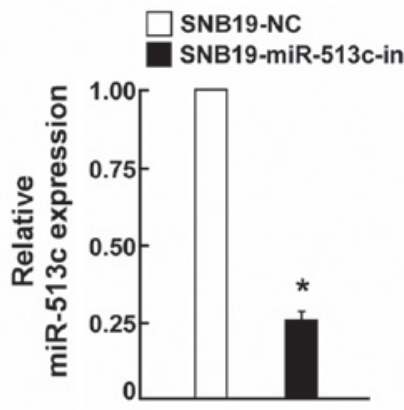

C

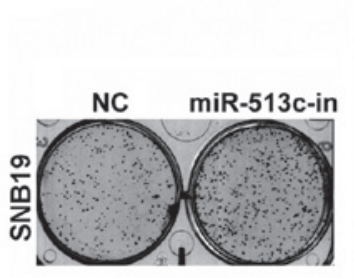

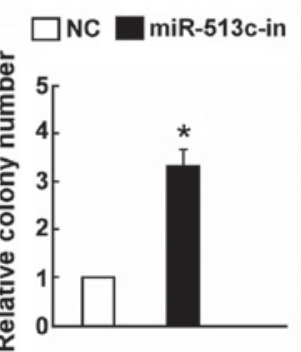

B

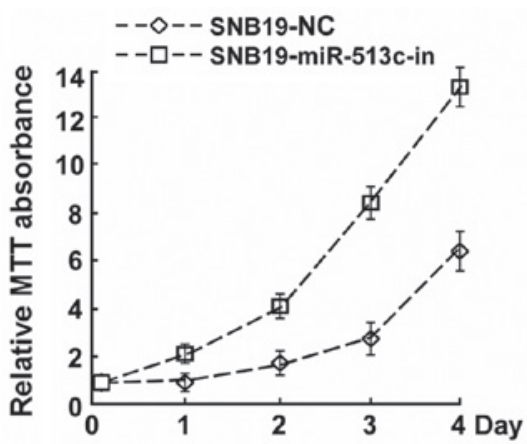

D
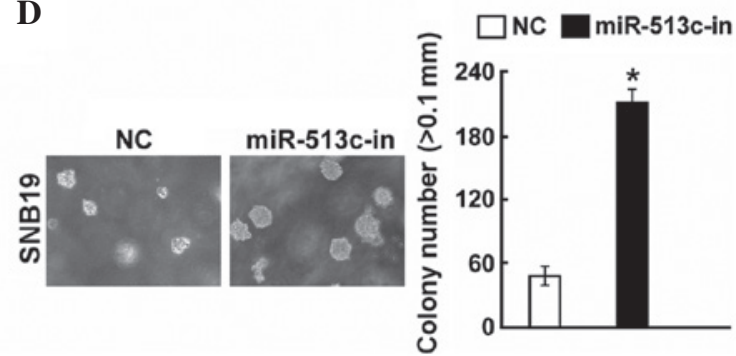

Figure 3. Inhibition of miR-513c promotes GBM cell proliferation. (A) Confirmation of the knockdown of miR-513c following transfection by reverse transcription quantitative polymerase chain reaction analysis. (B) MTT assays revealed that the inhibition of miR-513c promoted the growth of the SNB19 GBM cell line. (C) Representative micrographs (left) and quantification (right) of crystal violet-stained cell colonies (magnification, $\mathrm{x} 4$ ). (D) Inhibition of miR-513c promoted the anchorage-independent growth of SNB19 cells. Representative micrographs (left) and quantification of colonies, which were $>0.1 \mathrm{~mm}$ (right) (magnification, $\mathrm{x} 100)$. Values are expressed as the mean of three independent experiments ("P<0.05 vs. NC). GBM, glioblastoma; NC, negative control; miR, microRNA; $\mathrm{NC}$, normal control.

while miR-513c-in clearly promoted the protein expression of LRP6. To confirm the effect of miR-513c on the inhibition of the expression of LRP6, the present study assessed whether LRP6 was regulated by miR-513c through direct binding to its 3'UTR. A LRP6 3'-UTR wild-type vector was co-transfected into SNB19 cells with either the miR-513c mimic, miR-513c-in, miR-513c-mut or miR-NC, followed by measurement of luciferase activity. As shown in Fig. 4C, a consistent 


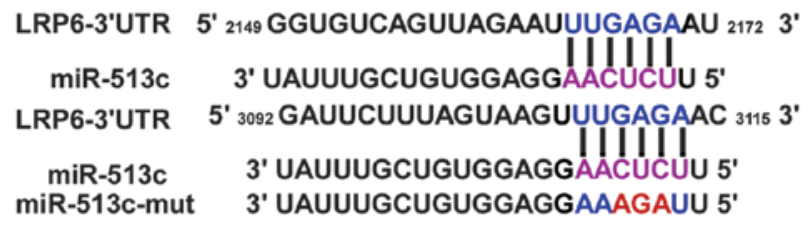

C

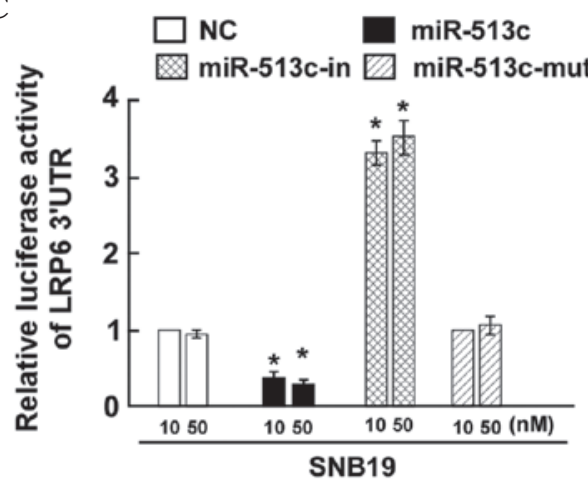

B

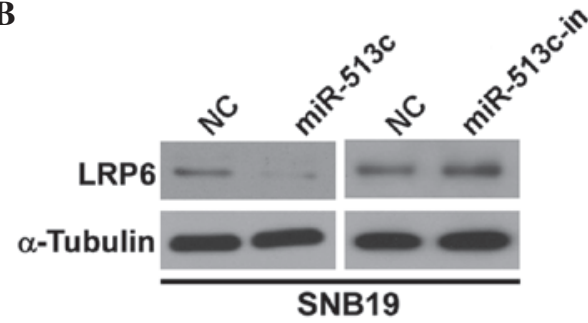

D

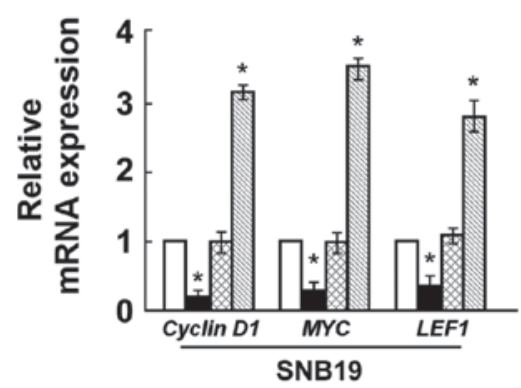

E

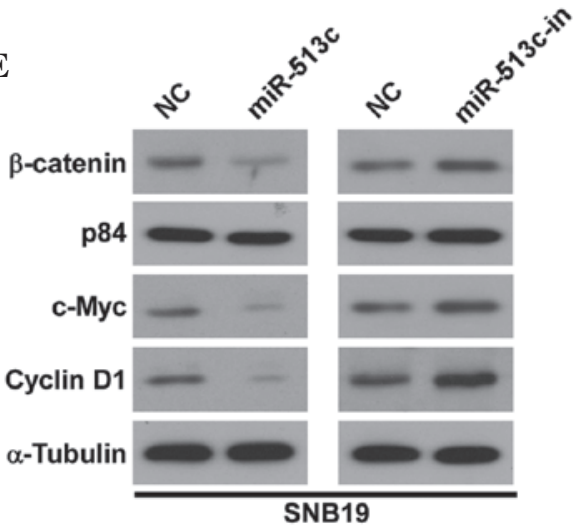

Figure 4. miR-513c suppresses the expression of LRP6 by directly targeting the LRP6 3'-UTR and alters the expression levels of proteins associated with proliferation or apoptosis in SNB19 cells. (A) The predicted miR-513c target sequence in the 3'-UTR of LRP6 (LRP6-3'-UTR) and the positions of three mutated nucleotides (red) in the 3'-UTR of miR-513C (miR-513c-mut). (B) Western blot analysis of the expression of LRP6 in cells transfected with miR-513c or the miR-513c inhibitor. $\alpha$-Tubulin was used as a loading control. (C) A luciferase reporter assay of the indicated cells transfected with the pGL3-LRP6-3'-UTR reporter and miR-513c or miR-513c-in with increasing quantitites (10 and $50 \mathrm{nM}$ ) of oligonucleotides. (D) Reverse transcription quantitative polymerase chain reaction analysis of the expression levels of cyclin D1, Myc and LEF1 in the indicated SNB19 cells. (E) Western blotting of the protein expression levels of $\beta$-catenin, p84, cyclin D1 and c-Myc in SNB19 cells. Nuclear protein p84 was used as a nuclear protein marker and $\alpha$-tubulin was used as the loading control ( $\mathrm{P}<0.05$ vs. NC). UTR, untranslated region; NC, negative control; LRP6, low-density lipoprotein receptor-related protein 6; NC, negative control; mut, mutant; miR-513c-in, microRNA-513c inhibitor.

B

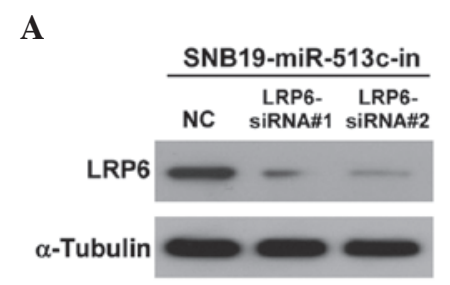

$\square$ SNB19-miR-513c-in+NC

SNB19-miR-513c-in+LRP6-siRNA\#1

SNB19-miR-513c-in+LRP6-siRNA\#2

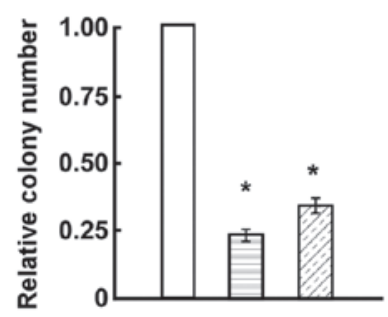

C

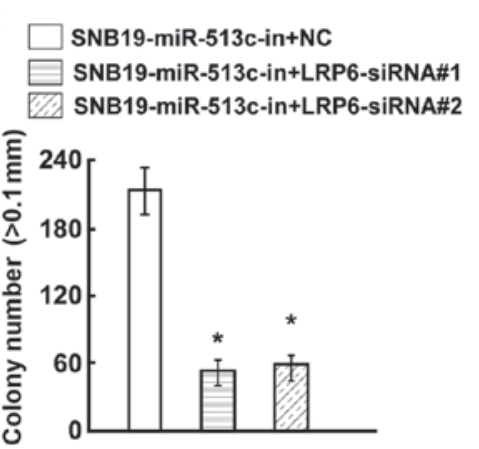

Figure 5. Upregulation of LRP6 is required for miR-513c-induced proliferation of GBM cells. (A) Representative quantification of crystal violet-stained cell colonies. (B) Inhibition of miR-513c in the LRP6-silenced cells revealed no additive effect to promote the anchorage-independent growth of SNB19 cells. (C) Representative quantification of colonies, which were $>0.1 \mathrm{~mm}$. Values are expressed as the mean of three independent experiments ( $\mathrm{P}<0.05 \mathrm{vs}$. NC). GBM, glioblastoma; LRP6, low-density lipoprotein receptor-related protein 6; NC, negative control; miR-513c-in, microRNA-513c inhibitor; siRNA, small interfering RNA. 
and dose-dependent reduction in the luciferase activity was observed in SNB19 cells transfected with the miR-513c mimic, whereas LRP6 luciferase activity was increased in the presence of miR-513c inhibitor. In addition, overexpression of miR-513c-mut had no significant effect on the luciferase activity of the LRP6 3'-UTR. These results demonstrated that LRP6 is a bona fide target of miR-513c.

miR-513c alters the levels of proteins associated with proliferation in GBM cells. The expression levels of a number of critical cell-proliferation regulators were also detected. It was previously reported that LRP6 is closely correlated with Wnt/ $\beta$ catenin signaling activity (6-8). The present study assessed the mRNA expression levels of the downstream genes in the Wnt/ $\beta$-catenin signaling pathway. The downstream genes regulated by Wnt/ $\beta$-catenin, cyclin D1, Myc and LEF1 were all significantly downregulated by miR-513c and upregulated by the miR-513c inhibitor (Fig. 4D). Furthermore, the protein expression levels of $\beta$-catenin, $c-M y c$ and cyclin D1 were reduced in SNB19 cells transfected with the miR-513c mimic, however, their expression was upregulated in the cells transfected with miR-513c-in compared with that in the control cells (Fig. 4E). These findings provided further evidence that miR-513c is important in GBM cell proliferation. These results indicated that miR-513c functionally modulates the cellular proliferation regulators c-Myc, LEF-1, cyclin D1 and $\beta$-catenin, and is therefore relevant to cell proliferation.

LRP6 upregulation is required for $m i R-513 c$-in-induced proliferation of GBM cells. To further investigate the role of LRP6 promotion in miR-513c-in-induced GBM proliferation, the effects of downregulation of LRP6 on GBM cell proliferation were assessed. As predicted, western blot analysis confirmed that LRP6-siRNA 1 and LRP6-siRNA ${ }^{\#} 2$ effectively decreased the expression levels of LRP6 in miR-513c-in-transfected SNB19 cells (Fig. 5A). Colony formation and anchorage-independent growth assays revealed that miR-513c-in in the LRP6-silenced cells had no additive effect on cell proliferation (Fig. 5B and C). These results demonstrated that direct upregulation of LRP6 was required for miR-513-in-induced GBM cell proliferation.

\section{Discussion}

miRNAs are a class of small(22 nucleotides), non-coding, highly conserved single-stranded RNAs with post-transcriptional regulatory features, and are important in multiple biological processes, including the regulation of cell proliferation, differentiation, survival and apoptosis $(3,9,10)$. The present study demonstrated that the expression levels of miR-513c were significantly downregulated in GBM tissues and GBM cells compared with those in the NHAs and normal brain tissues. Furthermore, ectopic expression of miR-513c decreased the growth rate and anchorage-independent growth of GBM cells, whereas miR-513c-in caused the opposite effect, indicating that miRNA-513c may function as a tumor suppressor miRNA. Through bioinformatic analysis, the LRP6 tumor promoter gene was identified as a theoretical miRNA-513c target gene. Western blot analysis demonstrated that ectopic miR-513c expression markedly reduced the protein expression levels of LRP6, whereas miR-513c-in clearly promoted the protein expression of LRP6. In addition, a luciferase reporter assay demonstrated that the downregulation of LRP6 was mediated by miR-513c through the LRP6 3'-UTR. These results demonstrated that LRP6 is a bona fide target of miR-513c.

LRP6 is a novel member of the expanding LDL receptor family, functions as an indispensable co-receptor and is critical in the activation of the canonical Wnt $/ \beta$-catenin signaling pathway (11-15). Wnt/ $\beta$-catenin signaling controls fundamental cellular processes and aberrant activation of this pathway is implicated in several types of human cancer (16-18). $\beta$-catenin is one of the major cellular effectors of the Wnt signaling pathway, which is essential in development and tissue maintenance (19). It is well known that the $\beta$-catenin signaling pathway is essential in the pathogenesis of various types of human cancer $(16,20,21)$. The present study aimed to further investigate the molecular mechanisms of growth inhibition induced by miR-513c. The expression of $\beta$-catenin was decreased in SNB19 cells transfected with the miR-513c mimic; however, was upregulated in the cells transfected with miR-513c-in. Furthermore, the expression levels of $\beta$-catenin downstream genes, including cyclin D1, Myc and LEF-1, were determined. CyclinD1, Myc and LEF-1 have been identified as target genes of the Wnt/ $\beta$-catenin signaling pathway, exerting a pivotal role in human cancer carcinogenesis (22-26). The present study demonstrated that the mRNA expression levels of these molecules were all significantly suppressed, suggesting that miR-513c may be an important regulator of this signaling pathway. Consistent with these observations, the protein expression levels of cyclin D1 and c-Myc were markedly upregulated following transfection with the miR-513c inhibitor and downregulated following overexpression of miR-513c. Furthermore, inhibition of miR-513c in the LRP6-silenced cells revealed no additive effect on cell proliferation, suggesting that direct upregulation of LRP6 was required for miR-513c-in-induced GBM cell proliferation.

In conclusion, the present study revealed that miR-513c is a tumor suppressor miRNA in GBM cells and that LRP6 is a direct target of miRNA-513c. Therefore, these findings indicated that miR-513c may serve as a potential therapeutic target for the treatment of GBM.

\section{Acknowledgements}

This study was supported by the Shandong Province Young and Middle-aged Scientists Research Awards Fund (no. BS200403017).

\section{References}

1. Wen PY and Kesari S: Malignant gliomas in adults. N Engl J Med 359: 492-507, 2008.

2. Baek D, Villén J, Shin C, Camargo FD, Gygi SP and Bartel DP: The impact of microRNAs on protein output. Nature 455: 64-71, 2008.

3. Bartel DP: MicroRNAs: Genomics, biogenesis, mechanism and function. Cell 116: 281-297, 2004.

4. Miska EA: How microRNAs control cell division, differentiation and death. Curr Opin Genet Dev 15: 563-568, 2005.

5. Ambros V: The functions of animal microRNAs. Nature 431: 350-355, 2004.

6. Hsu SY, Kudo M, Chen T, et al: The three subfamilies of leucine-rich repeat-containing $\mathrm{G}$ protein-coupled receptors (LGR): Identification of LGR6 and LGR7 and the signaling mechanism for LGR7. Mol Endocrinol 14: 1257-1271, 2000. 
7. Li Y, Lu W, He X, Schwartz AL and Bu G: LRP6 expression promotes cancer cell proliferation and tumorigenesis by altering beta-catenin subcellular distribution. Oncogene 23: 9129-9135, 2004.

8. Lu W, Lin C, King TD, Chen H, Reynolds RC and Li Y: Silibinin inhibits Wnt/beta-catenin signaling by suppressing Wnt co-receptor LRP6 expression in human prostate and breast cancer cells. Cell Signal 24: 2291-2296, 2012.

9. Formosa A, Markert EK, Lena AM, et al: MicroRNAs, miR-154, miR-299-5p, miR-376a, miR-376c, miR-377, miR-381, miR-487b, miR-485-3p, miR-495 and miR-654-3p, mapped to the 14q32.31 locus, regulate proliferation, apoptosis, migration and invasion in metastatic prostate cancer cells. Oncogene 33: 5173-5182, 2014.

10. Lehmann U, Streichert T, Otto B, et al: Identification of differentially expressed microRNAs in human male breast cancer. BMC Cancer 10: 109, 2010

11. Gong X, Carmon KS, Lin Q, Thomas A, Yi J and Liu Q: LGR6 is a high affinity receptor of R-spondins and potentially functions as a tumor suppressor. PLoS One 7: e37137, 2012.

12. Gray JD, Kholmanskikh S, Castaldo BS, et al: LRP6 exerts non-canonical effects on Wnt signaling during neural tube closure. Hum Mol Genet 22: 4267-4281, 2013.

13. Joiner DM, Ke J, Zhong Z, Xu HE and Williams BO: LRP5 and LRP6 in development and disease. Trends Endocrinol Metab 24: 31-39, 2013

14. Maletínská L, Blakely EA, Bjornstad KA, Deen DF, Knoff LJ and Forte TM: Human glioblastoma cell lines: levels of low-density lipoprotein receptor and low-density lipoprotein receptor-related protein. Cancer Res 60: 2300-2303, 2000

15. Semënov MV, Zhang X and He X: DKK1 antagonizes Wnt signaling without promotion of LRP6 internalization and degradation. J Biol Chem 283: 21427-21432, 2008.

16. Ochoa-Hernández AB, Juárez-Vázquez CI, Rosales-Reynoso MA and Barros-Núñez P: WNT- $\beta$-catenin signaling pathway and its relationship with cancer. Cir Cir 80: 389-398, 2012 (In Spanish).
17. $\mathrm{Kim} \mathrm{KH}$, Seol HJ, Kim EH, et al: Wnt/ $\beta$-catenin signaling is a key downstream mediator of MET signaling in glioblastoma stem cells. Neuro Oncol 15: 161-171, 2013.

18. Hsieh IS, Chang KC, Tsai YT, et al: MicroRNA-320 suppresses the stem cell-like characteristics of prostate cancer cells by downregulating the Wnt/beta-catenin signaling pathway. Carcinogenesis 34: 530-538, 2013.

19. Fu Y, Zheng S, An N, et al: $\beta$-catenin as a potential key target for tumor suppression. Int J Cancer 129: 1541-1551, 2011.

20. Chocarro-Calvo A, García-Martínez JM, Ardila-González S, De la Vieja A and García-Jiménez C: Glucose-induced $\beta$-catenin acetylation enhances Wnt signaling in cancer. Mol Cell 49: 474-486, 2013

21. Wang D, Wise ML, Li F and Dey M: Phytochemicals attenuating aberrant activation of $\beta$-catenin in cancer cells. PLoS One 7: e50508, 2012

22. He TC, Sparks AB, Rago C, et al: Identification of c-MYC as a target of the APC pathway. Science 281: 1509-1512, 1998.

23. Kolligs FT, Bommer G and Göke B: Wnt/beta-catenin/tcf signaling: a critical pathway in gastrointestinal tumorigenesis. Digestion 66: 131-144, 2002.

24. Nagayama M, Iwamoto M, Hargett A, et al: Wnt/beta-catenin signaling regulates cranial base development and growth. J Dent Res 87: 244-249, 2008.

25. Huang FI, Chen YL, Chang CN, Yuan RH and Jeng YM: Hepatocyte growth factor activates Wnt pathway by transcriptional activation of LEF1 to facilitate tumor invasion. Carcinogenesis 33: 1142-1148, 2012.

26. Planutiene M, Planutis $\mathrm{K}$ and Holcombe RF: Lymphoid enhancer-binding factor 1 , a representative of vertebrate-specific Lef1/Tcf1 sub-family, is a Wnt-beta-catenin pathway target gene in human endothelial cells which regulates matrix metalloproteinase-2 expression and promotes endothelial cell invasion. Vasc Cell 3: 28, 2011 